\title{
Recombinant adeno-associated virus-delivered anginex inhibits angiogenesis and growth of HUVECs by regulating the Akt, JNK and NF-kB signaling pathways
}

\author{
KE MA $^{1}$, CHUYING WANG ${ }^{1}$, QIANQIAN GENG ${ }^{2}$, YANGWEI FAN ${ }^{1}$, JING NING $^{1}$, HAIXIA YANG ${ }^{1}$, \\ XUYUAN DONG ${ }^{1}$, DANFENG DONG ${ }^{1}$, YUYAN GUO ${ }^{3}$, XIN WEI $^{4},{\text { ENXIAO } \mathrm{LI}^{1 *} \text { and YINYING WU }}^{1 *}$ \\ Departments of ${ }^{1}$ Medical Oncology, ${ }^{2}$ Nuclear Medicine and ${ }^{3}$ Medical Radiation Oncology, The First Affiliated Hospital \\ of Xi'an Jiaotong University, Xi'an, Shaanxi 710061; ${ }^{4}$ Department of Medical Oncology, \\ Shaanxi Province People's Hospital, Xi'an, Shaanxi 710068, P.R. China
}

Received December 1, 2015; Accepted January 5, 2016

DOI: 10.3892/or.2016.4711

\begin{abstract}
Anginex is an artificial synthetic small molecule $\beta$-sheet-forming peptide shown to have anti-angiogenesis and antitumor effects in various solid tumors. However, its molecular mechanism remains largely unclear and efficient delivery methods for anginex remains to be developed. We report on the development of recombinant adeno-associated virus (rAAV2)-delivered anginex and the underlying mechanism of anti-angiogenesis and antitumor effects of anginex. We have successfully developed the rAAV2 vector to efficiently express anginex (rAAV2-anginex). Transduction of rAAV2-anginex significantly induced apoptosis, and inhibited the proliferation, migration, invasion and tube formation of human umbilical vein endothelial cells in vitro. Western blot analysis revealed that rAAV2-anginex inhibited the phosphorylation of Akt, while inducing the phosphorylation of JNK and activation of the NF- $\kappa \mathrm{B}$ signaling pathway. In an in vivo CAM assay and xenograft model of SKOV3, rAAV2anginex significantly reduced microvessel density (MVD) and vascular endothelial growth factor $165\left(\mathrm{VEGF}_{165}\right)$, as demonstrated by immunohistochemistry analysis. Importantly, rAAV2-anginex inhibited tumor growth in an ovarian cancer SKOV3 cell nude mouse xenograft model. Our results suggest that rAAV2-anginex may inhibit tumor angiogenesis and growth through regulating Akt, JNK and $\mathrm{NF}-\kappa \mathrm{B}$ signaling pathways.
\end{abstract}

Correspondence to: $\mathrm{Dr}$ Yinying $\mathrm{Wu}$ or $\mathrm{Dr}$ Enxiao $\mathrm{Li}$, Department of Medical Oncology, The First Affiliated Hospital of Xi'an Jiaotong University, 277 Yanta West Road, Xi'an, Shaanxi 710061, P.R. China

E-mail: shadowless_111@163.com

E-mail: doclienxiao@163.com; makeyfly@126.com

*Contributed equally

Key words: anginex, peptide, angiogenesis, anti-angiogenesis, recombinant adeno-associated virus

\section{Introduction}

Angiogenesis is indispensable in cancer progression, and therefore a promising target for the development of cancer treatments. Endothelial cells (ECs) that line the tumor vasculature are ideal target cells for cancer therapeutics, since they are easily accessible to agents delivered by blood and are genetically stable without being easily mutated into drug-resistant variants $(1,2)$. Anginex, an antiparallel $\beta$-sheet-forming 33 -mer peptide, is structured and synthesized based on the basic folding principles and incorporation of short sequences from the $\beta$-sheet domains of natural anti-angiogenic agents including platelet factor-4 (PF4), interleukin-8 (IL-8) and bactericidal-permeability increasing protein 1 (BP1) (3-6). Anginex has been shown to prevent the proliferation, adhesion and migration of activated ECs, and induce apoptosis in these cells. Furthermore, it has been demonstrated that anginex significantly reduces tumor growth and angiogenesis in mouse models (7-9). These advances suggest that anginex is a very promising agent for the treatment of various solid tumors regardless of the heterogeneity of cancer cells.

As a small artificial synthetic peptide, anginex has the characteristics of hypotoxicity, hyperosmolality and high selectivity; but it can easily be hydrolyzed by endopeptidase with poor stability and short half-life. Recombinant anginex has been proven to have comparable angiostatic properties as the synthetic peptide (10). We have previously constructed the AdNT4-anginex virus, which secrete anginex and has shown apparent anti-angiogenesis and antitumor effects in vitro and in vivo $(11,12)$. However, adenovirus transduction is transient with a relatively strong host immune response (13). In contrast to adenovirus, adeno-associated virus (AAV) can deliver genes to cells to provide a stable expression with no immune response, and preferentially integrate into a specific site at the q arm of chromosome 19 (14-16). Therefore, AAV may be a more suitable vector to deliver anginex for the treatment of cancers. Previous studies have shown that anginex inhibits migration and proliferation, and induces apoptosis in ECs; however, its precise underlying molecular mechanism remains to be determined. 
In order to achieve a stable expression of anginex for cancer therapy, we constructed the recombinant adeno-associated virus (rAAV2)-anginex; and assessed the effects of rAAV2-anginex on the proliferation of human umbilical vein endothelial cells (HUVECs) in vitro, as well as its antiangiogenesis and antitumor effects in mouse tumor xenografts. Furthermore, we explored the mechanism of anti-angiogenesis and antitumor effects of rAAV2-anginex.

\section{Materials and methods}

Materials. The empty adeno-associated virus (AAV2) was purchased from Bo Miao Biotechnology (Beijing, China). The rAAV2-anginex virus was designed with NT4 secretion signal sequence at the $\mathrm{N}$-terminal side and the 6xHis-tag sequence at the C-terminal side of anginex. Titers of rAAV2-anginex and AAV2 virus were $4.4 \times 10^{11}$ and $5.0 \times 10^{11} \mathrm{vg} / \mathrm{ml}$, respectively. Four-week-old female athymic BALB/c nude mice were purchased from the Animal Center of the Medical College of Xi'an Jiaotong University. White leghorn eggs were purchased from Xi'an Chicken Breeding Station (Xi'an, China). Fetal bovine serum (FBS), Dulbecco's modified Eagle's medium (DMEM) and Rosewell Park Memorial Institute (RPMI)-1640 medium were purchased from HyClone (Logan, UT, USA). Vascular endothelial growth factor $165\left(\mathrm{VEGF}_{165} ; 100-20\right)$ was purchased from PeproTech (Rocky Hill, NJ, USA). NF- $\kappa$ B pathway sampler kit (\#9936), rabbit anti-His-tag monoclonal antibody (\#12698), rabbit anti-extracellular signal regulated kinase monoclonal antibody (ERK, \#4695), rabbit anti-phospho-ERK monoclonal antibody (p-ERK, \#4370), rabbit anti-phospho-Akt monoclonal antibody (p-Akt, \#4060), rabbit anti-Akt monoclonal antibody (\#4685), rabbit anti-phospho-c-Jun N-terminal kinase monoclonal antibody (p-JNK, \#4668), horseradish peroxidase (HRP)-conjugated anti-mouse (\#7046) and anti-rabbit (\#7044) IgG antibodies were purchased from Cell Signaling Technology (Danvers, MA, USA). Rabbit anti-CD31 polyclonal antibody (ab28364), rabbit anti-Bcl-2 monoclonal antibody (ab136285), rabbit anti-Bax polyclonal antibody (ab7977), rabbit anti-Fas polyclonal antibody (ab82419) and rabbit anti-cyclin D1 polyclonal antibody (ab7958) were purchased from Abcam (Cambridge, UK). GAPDH monoclonal antibody (HRP-60004), rabbit anti-VEGF $_{165}$ polyclonal antibody (19003-1-AP) and rabbit anti-JNK polyclonal antibody (51151-1-AP) were purchased from the Proteintech Group (Wuhan, China).

Cell culture. HUVECs and human ovarian cancer cell line SKOV3 were kindly provided by the Translational Medical Center of the Medical College of Xi'an Jiaotong University. HUVECs and SKOV3 cells were cultured at $37^{\circ} \mathrm{C}$ with $5 \% \mathrm{CO}_{2}$ in DMEM and RPMI-1640 medium, respectively, containing $10 \% \mathrm{FBS}, 100 \mathrm{U} / \mathrm{ml}$ of penicillin and $100 \mu \mathrm{g} / \mathrm{ml}$ of streptomycin (Life Technologies, Grand Island, NY, USA). HUVECs were transduced for $72 \mathrm{~h}$ with rAAV2-anginex or AAV2 virus at a multiplicity of infection (MOI) of $10^{6}$ for further experiments. Cells were passaged every 2-3 days in $100-\mathrm{mm}$ dishes and harvested at the end of the treatment period for further analysis.

MTT assay. HUVECs were plated into 96-well plates. The next day, cells were separately treated with rAAV2-anginex and
AAV2 virus, and medium as blank control. Each group with 7 repeats was incubated for 7 days. Cell viability was evaluated daily with [3-(4,5-dimethylthiazol-2-yl)-2,5-diphenyl tetrazolium bromide (MTT)] (5 mg/ml) assay (Promega, Shanghai, China). Briefly, $20 \mu \mathrm{l}$ of MTT was added and incubated in the dark for an additional $4 \mathrm{~h}$ at $37^{\circ} \mathrm{C}$. Then, the supernatant was discarded and $100 \mu 1$ of dimethyl sulfoxide (DMSO; SigmaAldrich, St. Louis, MO, USA) was added into each well to dissolve the formazan product. The absorbance of the enzyme was measured at $490 \mathrm{~nm}$ excitation emission wavelength using a microplate reader (Bio-Rad, Hercules, CA, USA).

EdU incorporation assay. Cell proliferation was assessed with 5-ethynyl-2'-deoxyuridine (EdU) incorporation assay (C10310-1; RiboBio, Guangzhou, China), according to the manufacturer's instructions. Briefly, HUVECs transduced with rAAV2-anginex and empty AAV2 virus, and untreated HUVECs were plated in triplicate in 96-well plates at a density of $3 \times 10^{3}$ cells/well, and cultured to logarithmic phase. Then, $25 \mu \mathrm{M}$ of EdU was added into each well and cells were cultured for an additional $1.5 \mathrm{~h}$ at $37^{\circ} \mathrm{C}$. Cells were fixed with $4 \%$ formaldehyde for $30 \mathrm{~min}$ at room temperature and treated with $0.5 \%$ Triton $\mathrm{X}-100$ for $10 \mathrm{~min}$ at room temperature for permeabilization. After washing with phosphate-buffered saline (PBS) 3 times, $100 \mu \mathrm{l}$ of 1X Apollo ${ }^{\circledR}$ reaction cocktail was added into each well; and cells were incubated in the dark for $30 \mathrm{~min}$ at room temperature. Then, cells were stained with $100 \mu \mathrm{l}$ of Hoechst 33342 in the dark for $30 \mathrm{~min}$ and visualized under a fluorescent microscope (Nikon-Eclipse; Nikon, Tokyo, Japan). EdU-positive (red) and Hoechst 33342-positive cells (blue) were counted using Image Pro Plus (IPP) 6.0 software (Media Cybernetics, Bethesda, MD, USA). EdU incorporation rate was expressed as the ratio of EdU-positive cells to total Hoechst 33342-positive cells.

Flow cytometric analysis for cell cycle and apoptosis. For cell cycle analysis, cells were trypsinized into single cell suspensions and fixed with $70 \%$ ethanol for $2 \mathrm{~h}$ at $4^{\circ} \mathrm{C}$. After washing with PBS, cells were treated with RNase A $(50 \mu \mathrm{g} / \mathrm{ml})$, and stained with $25 \mu \mathrm{g} / \mathrm{ml}$ of propidium iodide (PI) for $30 \mathrm{~min}$ at $37^{\circ} \mathrm{C}$ in the dark. Samples were analyzed using a flow cytometer (BD FACSCalibur; BD Biosciences, Franklin Lakes, NJ, USA) and data were analyzed with the ModiFit LT v2.0 software. For analysis of apoptosis, cells were harvested by trypsinization and washed with PBS, followed by double staining with PE Annexin V and 7-AAD, according to the manufacturer's instructions (559763; BD Biosciences).

Transwell invasion and migration assays. Each Transwell chamber with polycarbonate membrane filters with 24-well inserts and an $8-\mu \mathrm{m}$ pore size (Corning, Corning, NY, USA) was coated with $50 \mu \mathrm{l}$ of Matrigel (1:6 dilution; BD, San Diego, CA, USA), and the gel was polymerized at $37^{\circ} \mathrm{C}$ for $8 \mathrm{~h}$. Then, the prepared cells $\left(4 \times 10^{4}\right)$ were plated onto the upper chambers with serum-free medium (200 $\mu \mathrm{l} /$ well), while the bottom chambers were filled with DMEM containing $10 \%$ FBS $\left(600 \mu \mathrm{l} /\right.$ well). After incubation for $48 \mathrm{~h}$ at $37^{\circ} \mathrm{C}$ in $5 \%$ $\mathrm{CO}_{2}$, non-invading cells in the upper chamber were removed; and cells that invaded to the lower surface of the filter were fixed with $4 \%$ paraformaldehyde for $15 \mathrm{~min}$ and stained with 
crystal violet for $30 \mathrm{~min}$. The number of invasive cells were visualized under a microscope (Nikon-Eclipse) and quantified by counting the number of cells in five randomly chosen fields with 200-fold magnification. Migration assay was performed without adding Matrigel, and experimental steps were the same as the invasion assay; but the number of plated cells was $2 \times 10^{4}$.

Tube formation assay. Each well of the pre-chilled 96-well plate was bottom-coated with $50 \mu \mathrm{l}$ of Matrigel (BD) and incubated at $37^{\circ} \mathrm{C}$ for $30 \mathrm{~min}$ to polymerize. HUVECs transduced with the rAAV2-anginex and empty AAV2 virus, and untreated HUVECs were seeded into each well and incubated at $37^{\circ} \mathrm{C}$ with $5 \% \mathrm{CO}_{2}$ for $6 \mathrm{~h}$. Then, images were captured (magnification, $\mathrm{x} 40$ ) to evaluate tube formation using IPP 6.0 software (Media Cybernetics).

Chick chorioallantoic membrane (CAM) assay. CAM assay was performed as previously described (11). Briefly, fresh fertilized white leghorn eggs were incubated at $37^{\circ} \mathrm{C}$ with $60 \%$ relative humidity, and flipped every $4 \mathrm{~h}$ with an automatic hatching machine (Science Incubator, Shandong, China). On day 3 , a $1 \times 2 \mathrm{~cm}$ rectangular window was made to continually observe the angiogenesis of the chick embryo of the CAM, and this window was covered with tape to prevent dehydration during the non-observation time. On day 7, a piece of gelatin sponge was placed on the CAM for the convenience of intervention. On day 13, $1 \mu \mathrm{g}$ of $\mathrm{VEGF}_{165}+100 \mu \mathrm{l}$ of PBS, $1 \mu \mathrm{g}$ of $\mathrm{VEGF}_{165}+5.0 \times 10^{10} \mathrm{vg}$ of rAAV2-anginex and $1 \mu \mathrm{g}$ of $\mathrm{VEGF}_{165}+5.0 \times 10^{10} \mathrm{vg}$ of AAV2 were added, respectively, to the CAM. On day 18, CAM angiogenesis was monitored and photographed.

$R T-P C R$. Total RNAs were isolated using a Fast 200 RNA extraction kit (Feijie Biological Technology, Shanghai, China), while cDNA was synthesized with a reverse transcription kit (\#RR036A; Takara, Dalian, China), according to the manufacturer's instructions. PCR was performed using a PCR amplifying system (Bio-Rad). Primers were designed and synthesized by Sangon Biotech (Shanghai, China). Primer sequences of rAAV2-anginex were as follows: forward, 5'-GTA CGGTGGGAGGTCTATATAAGCA-3' and reverse, 5'-GGT CCCGGTGTCTTCTATGGA-3'; for an internal standard, primer sequences of GAPDH were as follows: forward, 5'-AGG TCCACCACTGACACGTT-3' and reverse, 5'-GCCTCAAG ATCAGCAAT-3'. The reaction consisted of a Premix Taq (12.5 $\mu \mathrm{l})$, forward and reverse primers $(0.4 \mu \mathrm{M}, 1 \mu \mathrm{l})$, cDNA $(1 \mu \mathrm{l})$ and $\mathrm{ddH}_{2} \mathrm{O}(10.5 \mu \mathrm{l})$. Reaction conditions were as follows: $94^{\circ} \mathrm{C}$ for $3 \mathrm{~min}, 25 \mathrm{cycles}$ of $94^{\circ} \mathrm{C}$ for $30 \mathrm{sec}, 59.6^{\circ} \mathrm{C}$ for $30 \mathrm{sec}$ and $72^{\circ} \mathrm{C}$ for $30 \mathrm{sec}$. PCR products were electrophoretically separated on $2 \%$ agarose gel, and bands were visualized by ethidium bromide staining. Band intensity was evaluated using an agarose gel imaging system (Bio-Rad).

Western blotting. HUVECs were lysed at $4^{\circ} \mathrm{C}$ for $30 \mathrm{~min}$ in cell lysis buffer containing protease and phosphatase inhibitors (Roche, Indianapolis, IN, USA). Lysates were centrifuged at $16,000 \mathrm{x}$ g for $20 \mathrm{~min}$ at $4^{\circ} \mathrm{C}$. Protein concentration of the supernatant was measured using a Pierce BCA protein assay kit (23227; Thermo Fisher Scientific, Waltham,
MA, USA), according to the manufacturer's instructions. A total of $80 \mu \mathrm{g}$ of each cell lysate was separated by $10 \%$ sodium dodecyl sulfate-polyacrylamide gel electrophoresis (SDS-PAGE) polyacrylamide gels and transferred onto a polyvinylidene difluoride (PVDF) membrane (Millipore Corp., Billerica, MA, USA). After blocking with 5\% skim milk in Tris-buffered saline (TBS) containing $0.1 \%$ Tween-20 at room temperature for $2 \mathrm{~h}$, the membranes were incubated with primary antibodies (diluted at $1: 1,000$ ) overnight at $4{ }^{\circ} \mathrm{C}$. GAPDH (diluted at 1:5,000) was used as a loading control. After washing 4 times in TBS/Tween-20, the membranes were incubated for $1 \mathrm{~h}$ at room temperature with goat anti-rabbit or horse anti-mouse HRP-conjugated antibody at 1:2,000 dilution in TBS/Tween-20 containing 5\% skim milk. After extensive washing in TBS/Tween-20, blots were visualized with an enhanced chemiluminescence kit (ECL; Millipore Corp.).

Xenograft model. A total of $1 \times 10^{7}$ SKOV3 cells in $100 \mu \mathrm{l}$ of PBS were subcutaneously injected into the left flank of female nude mice. When tumors reached an average size of $\sim 200 \mathrm{~mm}^{3}$, tumor-bearing mice were randomly divided into 3 groups $(n=6)$, and respectively treated with $\mathrm{AAAV} 2$-anginex $\left(1.8 \times 10^{11} \mathrm{vg}\right)$ and rAAV2 $\left(1.8 \times 10^{11} \mathrm{vg}\right)$, with PBS $(100 \mu \mathrm{l})$ as blank control. The two maximum vertical diameters (a, long diameter; b, short diameter) of the tumor were measured and recorded every other day. Mice were sacrificed after 30 days. Then, the final size of the tumor was measured and photographed. Tumor volumes were calculated by $0.5 \mathrm{xab}^{2}$. All experimental procedures were carried out according to protocols approved by the Ethics Committee for Animal Experimentation of the Medical College of Xi'an Jiaotong University, in accordance with the National Institutes of Health Guide for the Care and Use of Laboratory Animals.

Immunohistochemistry (IHC). Transplanted tumor tissues were formalin fixed, paraffin embedded and cut into $4-\mu \mathrm{m}$ serial sections. Expression of $\mathrm{VEGF}_{165}$ and microvessel density (MVD; CD31) was measured using IHC by a streptavidin-biotin peroxidase (SP) kit (SP-9001; Beijing Zhongshan Golden Bridge Biotechnology, Beijing, China), according to the manufacturer's instructions. Briefly, antigens were retrieved using a microwave at high power for $5 \mathrm{~min}$, followed by $13 \mathrm{~min}$ at middle-low power in $10 \mathrm{mM}$ of citrate buffer (pH 6.0). Then, the sections were treated with 3\% hydrogen peroxide in methanol for $10 \mathrm{~min}$ at room temperature to quench endogenous peroxidase activity, followed by incubation with reagent $\mathrm{A}$ for $15 \mathrm{~min}$ at room temperature. Subsequently, the sections were incubated with anti-CD31 polyclonal antibody (dilution, 1:50) and anti-VEGF ${ }_{165}$ polyclonal antibody (dilution, 1:200) in a humidified chamber at $4^{\circ} \mathrm{C}$ overnight. After washing in PBS, the sections were, respectively, incubated with reagent $\mathrm{B}$ and $\mathrm{C}$ for $15 \mathrm{~min}$ at $37^{\circ} \mathrm{C}$. Then, diaminobenzidine (ZLI-9018; Beijing Zhongshan Golden Bridge Biotechnology) was added to the sections, according to the manufacturer's instructions. Finally, the sections were rinsed with water and counterstained with Harris hematoxylin. For negative control, PBS was used instead of the primary antibody. The sections were observed and separately scored by two independent investigators. 

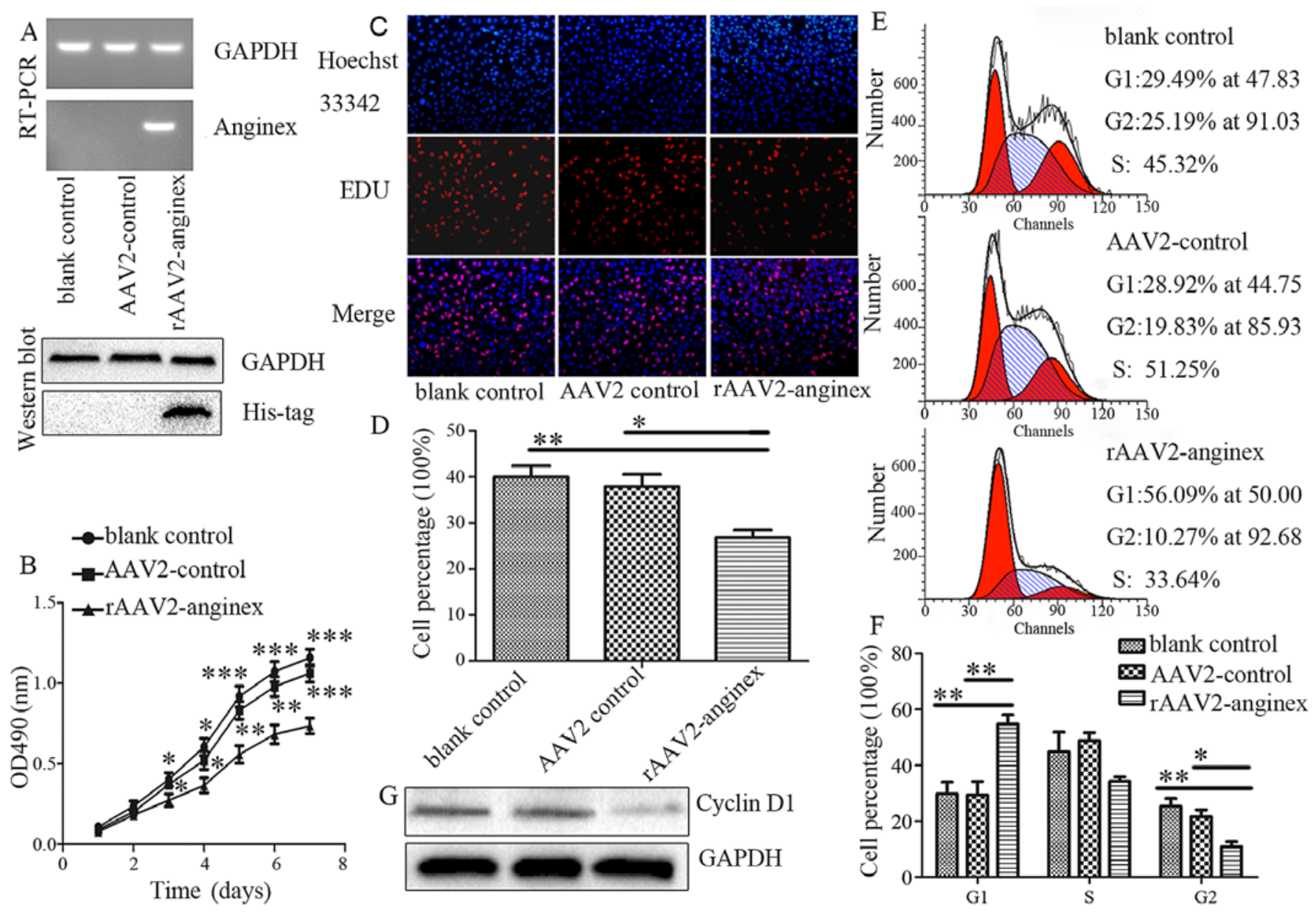

Figure 1. The rAAV2-anginex inhibits the proliferation of HUVECs. (A) RT-PCR (upper) and western blot analysis (lower) of anginex expression in HUVECs following transduction of rAAV2-anginex or AAV2. (B) Viability of HUVECs measured by MTT assay. (C and D) Images at a magnification of x200 (C) and corresponding histogram (D) results of the EDU incorporation assay; EDU-positive staining (red), Hoechst 33342-positive staining (blue). (E and F) Flow cytometric analysis of the HUVEC cell cycle, quantified in (F). Data are expressed as mean $\pm \mathrm{SEM}$. ${ }^{*} \mathrm{P}<0.05,{ }^{* * *} \mathrm{P}<0.01,{ }^{* * *} \mathrm{P}<0.001$, respectively, compared with the rAAV2-anginex group. (G) Expression levels of cyclin D1 was detected by western blot analysis. GAPDH was used as a loading control.

The MVD (CD31)-positivity judgment was based on the standard Weidner et al correction methods (17). Briefly, cytoplasmic staining for brown is positive staining; and both single cell and primmorph-positive staining, whether or not a lumen formation exists, are considered capillaries. Three concentrated areas as 'hot spots' at low magnification (x10) were randomly selected, and the number of capillaries in every hot area was counted at high magnification (x200). Results are expressed as mean \pm standard error (mean \pm SEM).

The expression intensity of $\mathrm{VEGF}_{165}$ was quantified as the sum of the integrated optical densities (IODs) of threshold pixels for all signals measured in each image. Then, the average value for each group was calculated. All imaging analysis was performed using IPP 6.0 software (Media Cybernetics). Results are expressed as mean \pm SEM.

Statistical analysis. Statistical analysis was performed using SPSS software (Statistical Package for the Social Sciences version 21.0; SPPS, Inc., Chicago, IL, USA). Differences between groups were analyzed by one-way ANOVA and LSD analysis. All statistical tests were two-sided. $\mathrm{P}<0.05$ was considered to indicate a statistically significant result.

\section{Results}

rAAV2-anginex inhibits HUVEC proliferation. Anginex targets activate ECs and potent antitumor and angiogenesis effects have been shown in mouse models (7-9). In order to achieve a stable expression of anginex for cancer therapy, a recombinant anginex adeno-associated virus, rAAV2anginex, was constructed. The expression of rAAV2-anginex was confirmed by RT-PCR ( $24 \mathrm{~h})$ and western blotting $(72 \mathrm{~h}$ ), respectively, after transduction of the rAA2-anginex and AAV2 virus in HUVECs. As shown in Fig. 1A, both the mRNA and protein of anginex were detected in rAAV2anginex virus-transduced cells. Next, the effect of anginex on HUVEC proliferation was assessed by MTT assay. HUVECs treated with the rAAV2-anginex virus grew slower from the third day $(\mathrm{P}=0.048)$, compared with the blank control group and empty virus AAV2. The inhibition effect was more obvious with increased treatment time, with a growth inhibition rate of $30.77 \%$ ( $\mathrm{P}<0.001$; Fig. 1B). EDU incorporation assay further verified that rAAV2-anginex significantly inhibited HUVEC proliferation $(\mathrm{P}=0.012$; Fig. $1 \mathrm{C}$ and $\mathrm{D})$. After incubation with EDU for $90 \mathrm{~min}$, EDU incorporation 

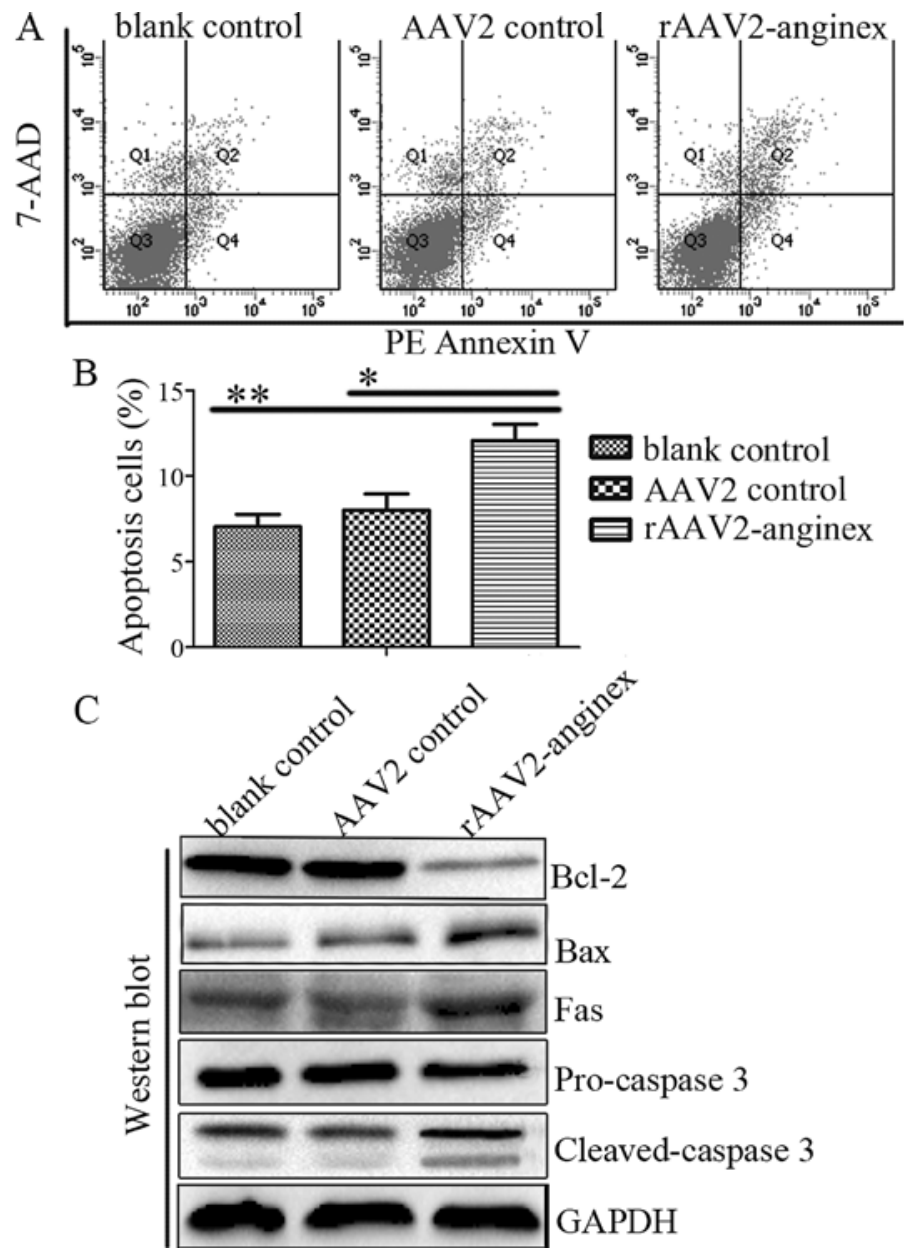

Figure 2. The rAAV2-anginex induces apoptosis in HUVECs. (A and B) Apoptosis of HUVECs was detected by flow cytometry with PE Annexin V and 7-AAD staining, quantified in (B). Data are expressed as mean \pm SEM; ${ }^{*} \mathrm{P}<0.05$ and ${ }^{* *} \mathrm{P}<0.01$, respectively, compared with the rAAV2-anginex group. (C) Expression levels of bcl-2, bax, fas, pro-caspase-3 and cleaved caspase-3 were detected by western blot analysis. GAPDH was used as a loading control.

in the rAAV2-anginex, blank control and AAV2 control group was $26.79 \pm 1.61 \%$ (mean \pm SEM), 39.62 $\pm 2.13 \%(\mathrm{P}<0.01)$ and $37.85 \pm 2.67 \%(\mathrm{P}<0.05)$, respectively. These results clearly show that rAAV2-anginex significantly inhibited HUVEC proliferation.

$\mathrm{PF} 4$, which is also an anti-angiogenic agent that contain $\beta$-sheet domains, has been shown to inhibit the cell cycle progression of ECs by blocking the transition from G1 to $S$ phase and the progression of $S$ phase (18). In order to test whether rAAV2-anginex alters cell cycle progression, flow cytometric analysis was carried out on HUVEC following the transduction of rAAV2-anginex. Compared with controls, rAAV2-anginex transduced cells displayed an increased percentage of cells in the G1 phase $(\mathrm{P}<0.01)$ and fewer cells in the $\mathrm{G} 2$ phase $(\mathrm{P}<0.05)$, but there was no significant difference in $\mathrm{S}$ phase distribution $(\mathrm{P}>0.05)$; implying that anginex may block the G1-S transition (Fig. 1E and F). Furthermore, western blot analysis revealed that rAAV2-anginex reduced cyclin D1 protein levels, compared with AAV2 (Fig. 1G). Taken together, these results demonstrate that rAAV2-anginex inhibited HUVEC proliferation by reducing the G1/S phase transition of HUVECs.

rAAV2-anginex induces HUVEC apoptosis. In order to explore whether rAAV2-anginex induces apoptosis,
HUVECs were stained with Annexin V, followed by flow cytometry. The transduction of rAAV2-anginex resulted in an elevated number of apoptotic cells, compared with the control groups $(\mathrm{P}<0.05$; Fig. $2 \mathrm{~A}$ and $\mathrm{B})$. Further immunoblotting revealed that $\mathrm{rAAV} 2$-anginex decreased $\mathrm{Bcl}-2$ protein levels and increased protein expression levels of Bax, Fas and cleaved-caspase-3 (Fig. 2C). These data indicate that rAAV2-anginex induced apoptosis in HUVECs.

rAAV2-anginex inhibits HUVEC migration and invasion. Previous studies have shown that anginex inhibited the adhesion and migration of ECs (19). Accordingly, the migration and invasion ability of HUVECs transduced with rAAV2-anginex were examined by Transwell migration and Matrigel invasion assays. Transwell migration assay revealed that rAAV2-anginex significantly decreased the migration of HUVECs, compared to the empty AAV2 and blank groups ( $\mathrm{P}<0.05$; Fig. $3 \mathrm{~A}$ and $\mathrm{B})$. In addition, Matrigel invasion assay demonstrated that rAAV2-anginex significantly reduced HUVEC invasion, compared to the empty AAV2 and blank group $(\mathrm{P}<0.05$; Fig. 3C and D). Thus, rAAV2-anginex had a remarkable inhibitory effect on HUVEC migration and invasion.

Modulation of Akt, JNK and NF- $\mathrm{B}$ signaling by rAAV2anginex in HUVECs. Akt, MAPK and NF- $\kappa \mathrm{B}$ signaling 


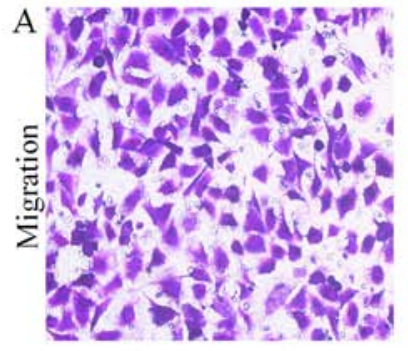

blank control

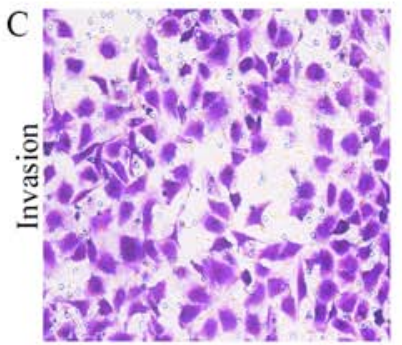

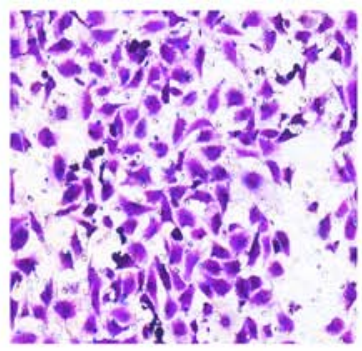

AAV2-control

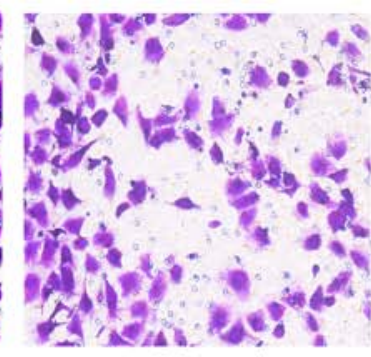

rAAV2-anginex

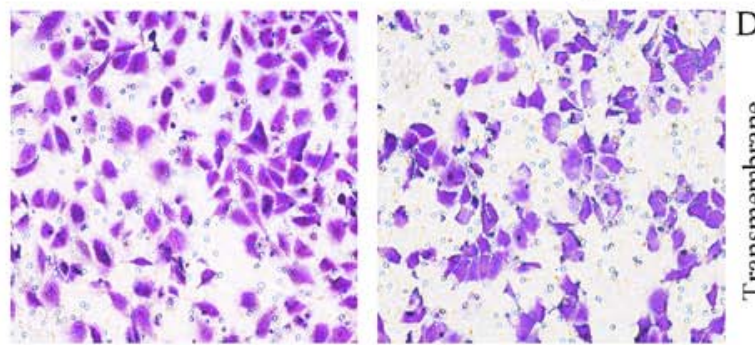

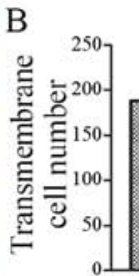

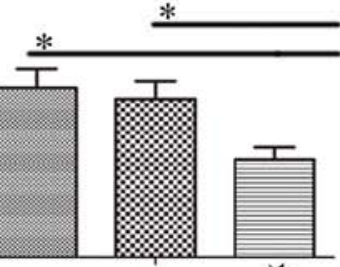

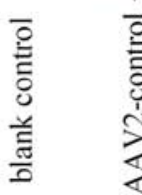

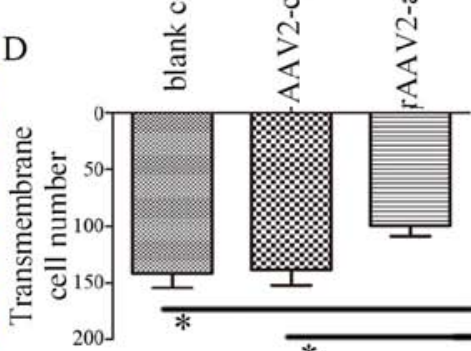

Figure 3. The rAAV2-anginex reduces the migration and invasion of HUVECs. (A and C) Images of HUVECs that migrated (upper) and invaded (lower) through the Transwell membrane for $48 \mathrm{~h}$, respectively, quantified in (B and D). Data are expressed as mean \pm SEM; ${ }^{*}<<0.05$, compared with the rAAV2-anginex group.

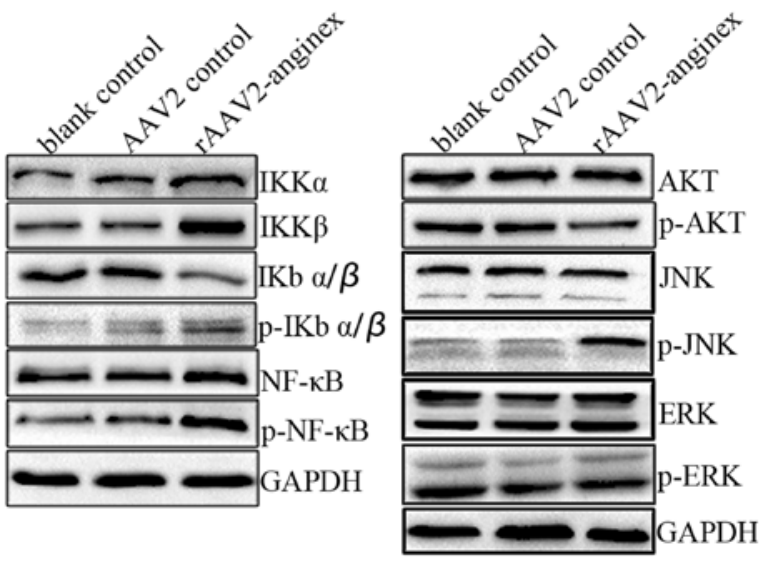

Figure 4. Effects of rAAV2-anginex on Akt and MAPK phosphorylation and the NF- $\kappa B$ signaling pathway in HUVECs. After treatment with rAAV2-anginex and AAV2 virus, the expression of IKK $\alpha$, IKK $\beta$, phosphorylated (p-) NF-kB, p-IKB $\alpha / \beta, p-A k t, p-J N K$ and p-ERK were examined by western blotting. GAPDH was used as loading control. Left to right: blank control group, AAV2 virus treatment and rAAV2-anginex virus treatment group.

pathways play an important role in the regulation of cell proliferation, survival, migration and invasion. In order to explore the potential mechanism for inhibiting cell proliferation, migration and invasion during rAAV2-anginex-induced HUVEC apoptosis, the activity of Akt, MAPK and the NF- $\mathrm{BB}$ signaling pathway was assessed by immunoblotting. As shown in Fig. 4, compared with the control group, p-Akt in the rAAV2-anginex treated group markedly decreased. For the MAPK signaling pathway, p-JNK increased in the rAAV2-anginex treated group, while p-ERK had no obvious change compared to the control group. In the NF- $\kappa \mathrm{B}$ signal pathway, for the expression of $\mathrm{p}-\mathrm{NF}-\kappa \mathrm{B}, \mathrm{p}-\mathrm{IKB} \alpha / \beta$ and IKK $\alpha, \operatorname{IKK} \beta$ increased and total IKB $\alpha / \beta$ decreased in the rAAV2-anginex group, suggesting that the $\mathrm{NF}-\kappa \mathrm{B}$ signaling pathway was activated by rAAV2-anginex. Thus, rAAV2-anginex treatment resulted in the inhibition of Akt and activation of the JNK and NF- $\kappa \mathrm{B}$ signaling pathways, but not ERK signaling in HUVECs.

rAAV2-anginex inhibits angiogenesis in HUVECs and chick embryo. In order to assess the anti-angiogenesis effect of rAAV2-anginex, tube formation assay in vitro and CAM assay in vivo, were performed. Compared with blank control and AAV2 control, rAAV2-anginex-treated HUVECs formed incomplete tube-like structures; and the extent of the tube formations of HUVECs significantly decreased $(\mathrm{P}<0.05$; Fig. 5A and B). In the in vivo CAM assay, rAAV2-anginex treatment significantly resulted in a decreased number of blood vessels and even the disappearance of blood vessels in some areas. Compared with vessels in the PBS group and AAV2 group, the rAAV2-anginex-treated group revealed that the caliber, distribution and length of vessels were reduced. Moreover, in the rAAV2-anginex treated group, coarse vessels became discontinued and obliterated, and the vascular net resembling leaf vein vanished and was replaced by confused arrangement and disfiguration (Fig. 5C). Therefore, rAAV2anginex significantly inhibited angiogenesis both in HUVECs in vitro and chick embryo in vivo.

rAAV2-anginex reduces angiogenesis and inhibits tumor growth in ovarian cancer SKOV3 cell nude mice xenograft model. In order to further explore the anti-angiogenesis and antitumor effects of rAAV2-anginex, an ovarian cancer SKOV3 cell nude mice xenograft model was applied to determine the anti-angiogenesis and antitumor effects of rAAV2-anginex. When the subcutaneous tumor volume reached $\sim 200 \mathrm{~mm}^{3}$, mice were treated with PBS, AAV2 and rAAV2-anginex. On day 8 after treatment, transplanted tumor volume in the rAAV2anginex group was significantly lower than in the control groups $(\mathrm{P}<0.05)$. On the last day of observation (day 30$)$, inhibition rates of tumor growth in the rAAV2-anginex group were $43.20 \%(\mathrm{P}<0.01)$ and $46.56 \%(\mathrm{P}<0.01)$ relative to the PBS 

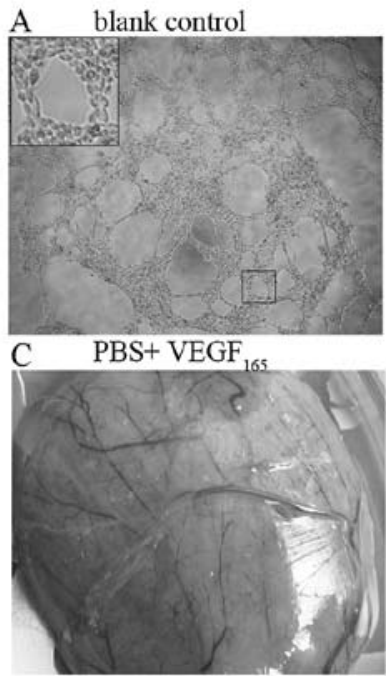

AAV2- control

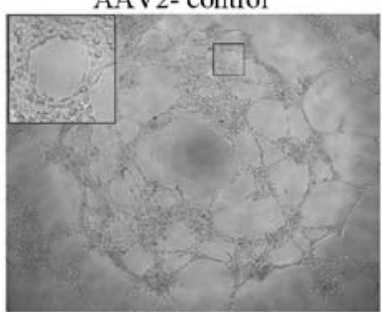

$\mathrm{AAV}_{2}+\mathrm{VEGF}_{165}$

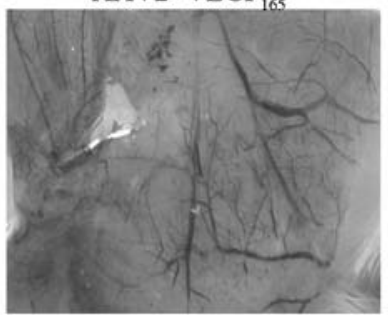

rAAV2-anginex
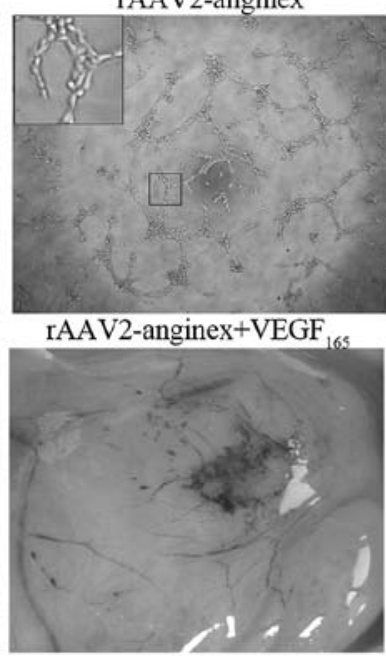

B Tube formation area (pixel)

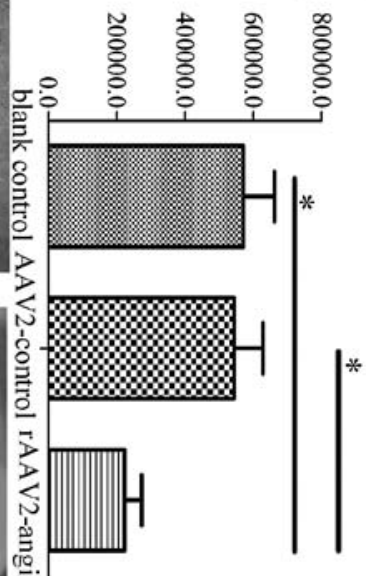

Figure 5. Anti-angiogenesis effect of rAAV2-anginex on HUVECs and in chick embryo CAM. (A and B) The capability of HUVECs to form a capillary-like network was observed after $72 \mathrm{~h}$ of rAAV2-anginex or AAV2 pre-treatment (magnification, $\mathrm{x} 40$ ), quantified in (B). Data are expressed as mean \pm SEM; "P $<0.05$, compared with the rAAV2-anginex group. (C) CAM assay revealed that in the rAAV2-anginex-treated group, the quantity, caliber and length of vessels were significantly reduced and even discontinued, which demonstrate that rAAV2-anginex inhibited the development of vasculature in chick embryo CAM.
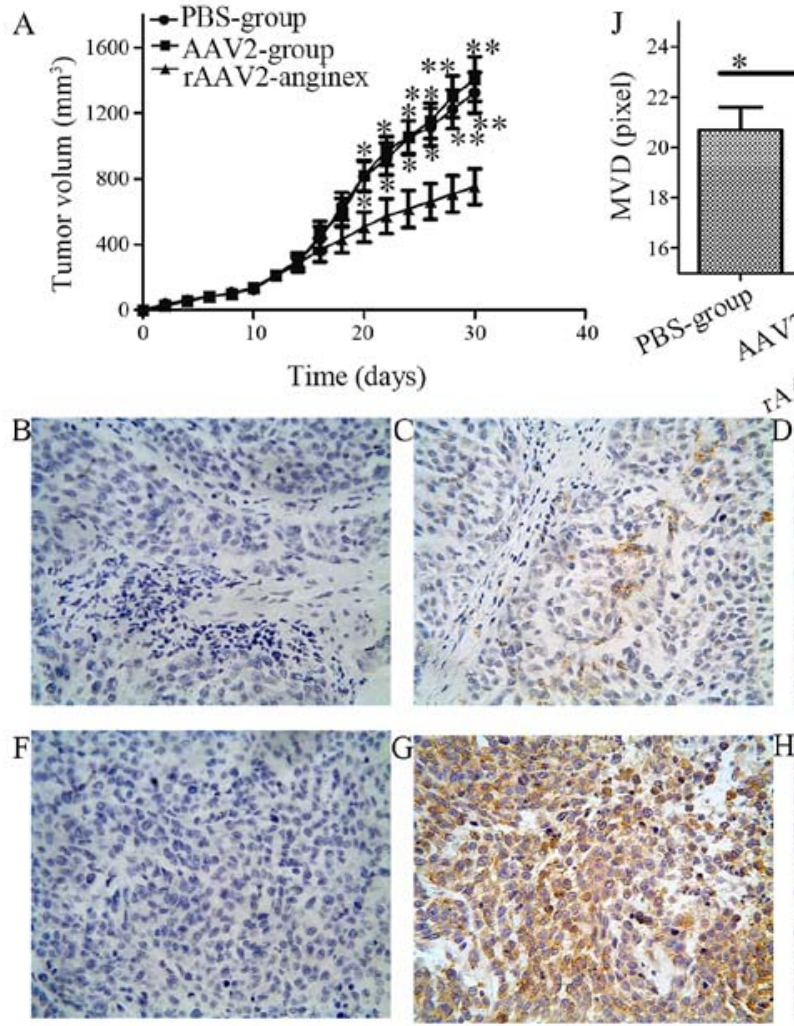
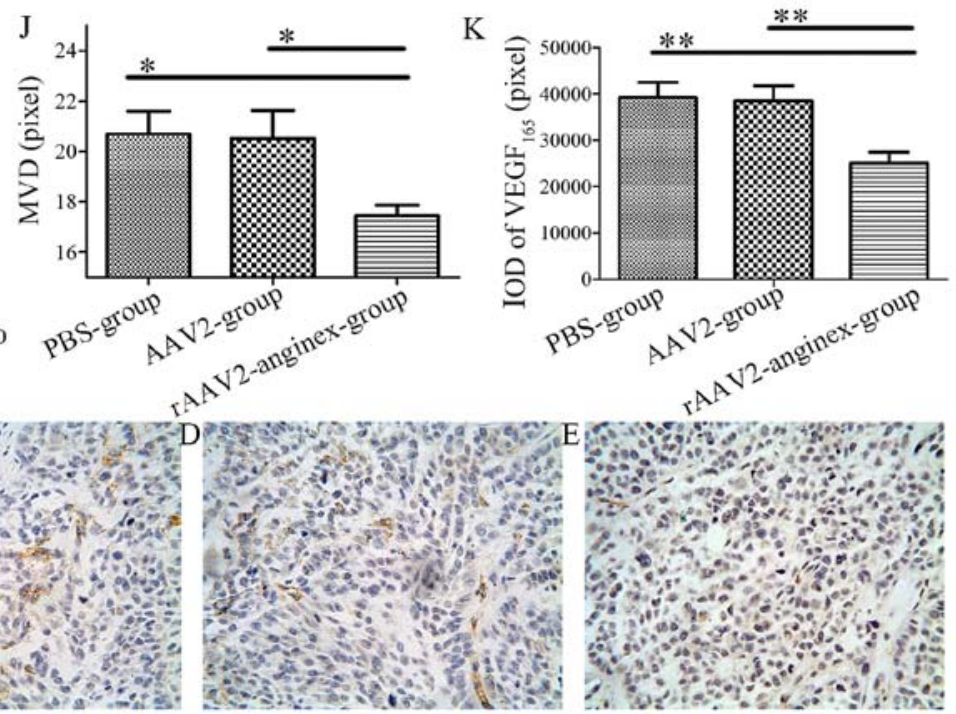

Figure 6. Anti-angiogenesis and antitumor effects of rAAV2-anginex in vivo. (A) The transplantation of tumor volumes was monitored regularly in SKOV3 xenograft models. Data are expressed as mean $\pm \mathrm{SEM}$; ${ }^{*} \mathrm{P}<0.05$ and ${ }^{* *} \mathrm{P}<0.01$, respectively, compared with the rAAV2-anginex group. (B-G) Representative immunohistochemical staining of MVD (CD31) (upper, B-E) and VEGF $_{165}$ (lower, F-I) in nude mouse xenograft tissues of human ovarian cancer cell line SKOV3. (B) Immunostaining for PBS (magnification, $\mathrm{x} 400$ ). (C) Immunostaining for CD31 in the PBS-treated group (magnification, $\mathrm{x} 400$ ). (D) Immunostaining for CD31 in the AAV2-treated group (magnification, x400). (E) Immunostaining for CD31 in the rAAV2-anginex treated group (magnification, 400). (F) Immunostaining for PBS (magnification, 400). (G) Immunostaining for VEGF 165 in the PBS-treated group (magnification, 400). (H) Immunostaining for $\mathrm{VEGF}_{165}$ in the AAV2-treated group (magnification, 400). (I) Immunostaining for $\mathrm{VEGF}_{165}$ in the rAAV2-anginex-treated group (magnification, 400). (J) Qualified of MVD (CD31). (K) Qualified IOD of VEGF ${ }_{165}$.

and AAV2 groups, respectively (Fig. 6A). However, there were no significant differences in food and water intake, exercise scope and intensity, and response sensitivity in mice among the groups, suggesting the safety of the adeno-associated virus. 
In order to test the anti-angiogenesis effect of rAAV2anginex in mice, $\mathrm{VEGF}_{165}$ and MVD were assessed by IHC. CD31 is marker of vascular endothelial cells, and the IHC of CD31 was used for MVD (Fig. 6B-E). According to the Weidner et al (17) correction method, MVD values were significantly different among the 3 groups $(P<0.05$, Fig. $6 \mathrm{~J})$. Mean MVD value in the rAAV2-anginex group was $17.45 \pm 0.42$ (mean \pm SEM), which significantly decreased compared with the AAV2 group $(20.52 \pm 1.12 ; \mathrm{P}=0.024)$ and PBS group (20.70 $\pm 0.91 ; P=0.018)$; and there was no statistically significant difference in MVD values between the AAV2 and PBS group $(\mathrm{P}=0.885)$. These results revealed that rAAV2-anginex significantly inhibited the microvascular density of ovarian transplantation tumor tissues.

Immunostaining of $\mathrm{VEGF}_{165}$ was observed on the plasma membrane and in the cytoplasm (Fig. 6F-I). Mean IOD of paraffin sections in the rAAV2-anginex group was $25,118.33 \pm 2,351.43$ (mean \pm SEM), which was significantly lower than the PBS group $(39,208.00 \pm 3,256.43 ; \mathrm{P}<0.01)$ and AAV2 group $(38,521.17 \pm 3,260.06 ; \mathrm{P}<0.01$; Fig. $6 \mathrm{I})$. There was no statistically significant difference in the IOD of paraffin sections between the AAV2 and PBS group $(\mathrm{P}=0.873)$. Thus, the rAAV2-anginex virus significantly inhibited the protein expression level of $\mathrm{VEGF}_{165}$ in ovarian transplantation tumors.

\section{Discussion}

In the present study, we demonstrated that the rAAV2-anginex virus significantly inhibited the proliferation, migration and invasion of HUVECs, accompanied with cell cycle arrest at the G1/S phase and induction of apoptosis. Moreover, rAAV2-anginex treatment led to the inhibition of Akt and activation of the $\mathrm{JNK}$ and $\mathrm{NF}-\kappa \mathrm{B}$ signaling pathways in HUVECs. Furthermore, rAAV2-anginex significantly inhibited angiogenesis both in HUVECs in vitro and chick embryo in vivo. Importantly, rAAV2-anginex inhibited angiogenesis and tumor growth in the ovarian cancer SKOV3 cell nude mouse xenograft model.

Anginex is a small artificial cytokine-like $\beta$-sheet-forming peptide with anti-angiogenesis and antitumor effects. However, its poor stability and short half-life limits its further development for cancer therapy. In the present study, we applied the recombinant adeno-associated virus (rAAV) as a delivery method for anginex (rAAV2-anginex). We found that rAAV2-anginex significantly inhibited G1/S phase transition, accompanied by the downregulation of cyclin D1. Cyclin D1 is a downstream target of PI3K-Akt signaling, which is a predominant cell growth-promoting signaling pathway (20). Notably, rAAV2-anginex significantly reduced the phosphorylation of Akt. Furthermore, Akt signaling indirectly and directly regulates pro-apoptosis and anti-apoptosis proteins, and ultimately blocks the apoptosis process (21-23). In the present study, we found that rAAV2-anginex significantly induced apoptosis, which was accompanied by the increase in pro-apoptosis proteins Bax and Fas and decrease of anti-apoptosis protein $\mathrm{Bcl}-2$. These results indicate that the inhibition of Akt signaling is one of the mechanisms by which rAAV2-anginex inhibited proliferation and induced apoptosis in HUVECs. Akt signaling had been reported to activate NF- $\mathrm{B}$ signaling (24). The activation of NF- $\mathrm{B}$ signaling in endothelial cells is critical for the activity of angiostatic agents including anginex (25). However, in the present study, rAAV2-anginex inhibited Akt signaling and activated the $\mathrm{NF}-\kappa \mathrm{B}$ signaling pathway, suggesting that rAAV2-anginex may induce apoptosis and inhibit angiogenesis through different signaling pathways. Surprisingly, we found that rAAV2-anginex induced phosphorylation of JNK. Since NF- $\mathrm{B}$ and JNK signaling are two key regulators of the pathophysiology of cells and a wide range of cross-talk occurs between these two signaling pathways (26-28), it is possible that the activation of the JNK/NF- $\mathrm{BB}$ signaling pathway may be involved in the antitumor and anti-angiogenesis effects of rAAV2-anginex. Additionally, galectin-1 is a cell surface receptor that promotes H-Ras signaling to the Raf/mitogenactivated protein kinase/extracellular signal regulated kinase (Erk) kinase (Mek)/Erk cascade. It has been reported that synthetic anginex could bind galectin-1 in activated ECs and disrupt the adhesion and migration of tumor ECs, thereby resulting in the inhibition of tumor angiogenesis (29). However, in the present study, rAAV2-anginex did not significantly change the total expression of Erk and phospho-Erk, suggesting that rAAV2-anginex may inhibit angiogenesis independent of Erk signaling.

Considering the potent effects of rAAV2-anginex on HUVECs in vitro, we further explored the anti-angiogenesis and antitumor growth effects of rAAV2-anginex in vivo; and found that rAAV2-anginex significantly inhibited angiogenesis in chick embryo. Importantly, the xenograft model further confirmed the safety and efficiency of rAAV2-anginex for further clinical trials. Compared with the PBS and AAV2 group, inhibition rates of tumor growth in the rAAV2-anginex group were 43.20 and $46.56 \%$ on the day 30 ; which is less than that reported in the (73\%) B16F10 xenograft (7) and (80\%) MA148 xenograft models (9) of synthetic anginex. These different inhibitory effects may be due to the different xenograft models chosen, and the observation times. In addition, the synthetic anginex was continuously injected for 21 or 28 days; while in the present study, the rAAV2-anginex virus was injected only once, which may have influenced tumor growth to some extent. In the study of Brandwijk et al (30), recombinant and synthetic anginex injected every 3 days inhibited tumor growth by $\sim 80$ and 55\%, respectively; which demonstrated the remarkable antitumor effect of recombinant anginex. Surprisingly, the decreased expression of $\mathrm{VEGF}_{165}$ protein in the rAAV2anginex virus-treated group may provide a new understanding of the anti-angiogenesis of rAAV2-anginex.

In summary, the present study demonstrates that rAAV2-anginex inhibited the proliferation, migration and invasion of HUVECs, as well as promoted apoptosis of HUVECs, ultimately leading to the inhibition of angiogenesis and tumor growth. Additionally, the xenograft model revealed that the adeno-associated virus is safe and has a long-lasting effect, providing a new treatment option for cancer therapy. More importantly, the present study indicates that the regulation of the Akt, JNK and NF- $\mathrm{NB}$ signaling pathways are potential mechanisms of the effects of rAAV2-anginex on HUVECs.

\section{Acknowledgements}

The present study was supported by grants from the National Natural Science Foundation of China (no. 81172169). 


\section{References}

1. Griffioen AW and Molema G: Angiogenesis: Potentials for pharmacologic intervention in the treatment of cancer, cardiovascular diseases, and chronic inflammation. Pharmacol Rev 52: 237-268, 2000.

2. Folkman J: Angiogenesis in cancer, vascular, rheumatoid and other disease. Nat Med 1: 27-31, 1995.

3. Mayo KH, Haseman J, Ilyina E and Gray B: Designed beta-sheet-forming peptide 33 mers with potent human bactericidal/permeability increasing protein-like bactericidal and endotoxin neutralizing activities. Biochim Biophys Acta 1425: 81-92, 1998.

4. Griffioen AW, van der Schaft DW, Barendsz-Janson AF, Cox A, Struijker Boudier HA, Hillen HF and Mayo KH: Anginex, a designed peptide that inhibits angiogenesis. Biochem J 354: 233-242, 2001.

5. Ilyina E, Roongta $\mathrm{V}$ and Mayo KH: NMR structure of a de novo designed, peptide 33mer with two distinct, compact beta-sheet folds. Biochemistry 36: 5245-5250, 1997.

6. Dings RP, Arroyo MM, Lockwood NA, van Eijk LI, Haseman JR, Griffioen AW and Mayo KH: Beta-sheet is the bioactive conformation of the anti-angiogenic anginex peptide. Biochem J 373 281-288, 2003.

7. van der Schaft DW, Dings RP, de Lussanet QG, van Eijk LI, Nap AW, Beets-Tan RG, Bouma-Ter Steege JC, Wagstaff J, Mayo KH and Griffioen AW: The designer anti-angiogenic peptide anginex targets tumor endothelial cells and inhibits tumor growth in animal models. FASEB J 16: 1991-1993, 2002.

8. Mayo KH, van der Schaft DW and Griffioen AW: Designed betasheet peptides that inhibit proliferation and induce apoptosis in endothelial cells. Angiogenesis 4: 45-51, 2001.

9. Dings RP, van der Schaft DW, Hargittai B, Haseman J, Griffioen AW and Mayo KH: Anti-tumor activity of the novel angiogenesis inhibitor anginex. Cancer Lett 194: 55-66, 2003.

10. Brandwijk RJ, Nesmelova I, Dings RP, Mayo KH, Thijssen VL and Griffioen AW: Cloning an artificial gene encoding angiostatic anginex: From designed peptide to functional recombinant protein. Biochem Biophys Res Commun 333: 1261-1268, 2005.

11. Dong DF, Li EX, Wang JB, Wu YY, Shi F, Guo JJ, Wu Y, Liu JP, Liu SX and Yang GX: Anti-angiogenesis and anti-tumor effects of AdNT4-anginex. Cancer Lett 285: 218-224, 2009.

12. Li EX, Liu SX, Yang GX, Wang QY, Wu YY and Shi F: Construction and identification of the recombinant prokaryotic expression plasmid containingbetapep-25 peptide. Xi Bao Yu Fen Zi Mian Yi Xue Za Zhi 22: 154-160, 2006 (In Chinese).

13. Berns KI and Giraud C: Adenovirus and adeno-associated virus as vectors for gene therapy. Ann NY Acad Sci 772: 95-104, 1995.

14. Samulski RJ, Zhu X, Xiao X, Brook JD, Housman DE, Epstein N and Hunter LA: Targeted integration of adeno-associated virus (AAV) into human chromosome 19. EMBO J 10: 3941-3950, 1991.

15. Kotin RM, Menninger JC, Ward DC and Berns KI: Mapping and direct visualization of a region-specific viral DNA integration site on chromosome 19q13-qter. Genomics 10: 831-834, 1991.
16. Kotin RM, Linden RM and Berns KI: Characterization of a preferred site on human chromosome $19 \mathrm{q}$ for integration of adeno-associated virus DNA by non-homologous recombination. EMBO J 11: 5071-5078, 1992.

17. Weidner N, Semple JP, Welch WR and Folkman J: Tumor angiogenesis and metastasis - correlation in invasive breast carcinoma. N Engl J Med 324: 1-8, 1991.

18. Gupta SK and Singh JP: Inhibition of endothelial cell proliferation by platelet factor- 4 involves a unique action on $\mathrm{S}$ phase progression. J Cell Biol 127: 1121-1127, 1994

19. Wang JB, Wang MD, Li EX and Dong DF: Advances and prospects of anginex as a promising anti-angiogenesis and antitumor agent. Peptides 38: 457-462, 2012.

20. Muise-Helmericks RC, Grimes HL, Bellacosa A, Malstrom SE, Tsichlis PN and Rosen N: Cyclin D expression is controlled post-transcriptionally via a phosphatidylinositol 3-kinase/Akt-dependent pathway. J Biol Chem 273: 29864-29872, 1998.

21. Xin M and Deng X: Nicotine inactivation of the proapoptotic function of Bax through phosphorylation. J Biol Chem 280: 10781-10789, 2005.

22. Henshall DC, Araki T, Schindler CK, Lan JQ, Tiekoter KL, Taki W and Simon RP: Activation of Bcl-2-associated death protein and counter-response of Akt within cell populations during seizure-induced neuronal death. J Neurosci 22: 8458-8465, 2002.

23. Datta SR, Brunet A and Greenberg ME: Cellular survival: A play in three Akts. Genes Dev 13: 2905-2927, 1999.

24. Pianetti S, Arsura M, Romieu-Mourez R, Coffey RJ and Sonenshein GE: Her-2/neu overexpression induces NF-kappaB via a PI3-kinase/Akt pathway involving calpain-mediated degradation of IkappaB-alpha that can be inhibited by the tumor suppressor PTEN. Oncogene 20: 1287-1299, 2001.

25. Tabruyn SP, Mémet S, Avé P, Verhaeghe C, Mayo KH, Struman I, Martial JA and Griffioen AW: NF-kappaB activation in endothelial cells is critical for the activity of angiostatic agents. Mol Cancer Ther 8: 2645-2654, 2009.

26. Karin M: Nuclear factor-kappaB in cancer development and progression. Nature 441: 431-436, 2006.

27. Chen F, Castranova V and Shi X: New insights into the role of nuclear factor-kappaB in cell growth regulation. Am J Pathol 159: 387-397, 2001.

28. Liu J and Lin A: Wiring the cell signaling circuitry by the NF-kappa B and JNK1 crosstalk and its applications in human diseases. Oncogene 26: 3267-3278, 2007.

29. Thijssen VL, Postel R, Brandwijk RJ, Dings RP, Nesmelova I, Satijn S, Verhofstad N, Nakabeppu Y, Baum LG, Bakkers J, et al: Galectin-1 is essential in tumor angiogenesis and is a target for antiangiogenesis therapy. Proc Natl Acad Sci USA 103: 15975-15980, 2006.

30. Brandwijk RJ, Dings RP, van der Linden E, Mayo KH, Thijssen VL and Griffioen AW: Anti-angiogenesis and antitumor activity of recombinant anginex. Biochem Biophys Res Commun 349: 1073-1078, 2006. 